\title{
Experimental human hookworm infection: therapeutic potential
}

This article was published in the following Dove Press journal:

Reports in Parasitology

12 August 2016

Number of times this article has been viewed

\section{Soraya T Gaze}

Cellular and Molecular Immunology Laboratory, Centro de Pesquisas Rene Rachou - Oswaldo Cruz Foundation, Belo Horizonte, Minas Gerais, Brazil
Correspondence: Soraya T Gaze Cellular and Molecular Immunology Laboratory, Centro de Pesquisas Rene Rachou - Oswaldo Cruz Foundation, Avenue Augusto de Lima 1715, 30.190002 Belo Horizonte, Minas Gerais, Brazil Tel +55 3l 33497779

Email soraya.gaze@cpqrr.fiocruz.br
Abstract: There has been an increase in the number of autoimmune and inflammatory diseases around the world, especially in developing countries. Helminths are masters of inducing an immune response that could potentially overcome those generated by unrelated host diseases. Hence, it is no surprise that there are studies focusing on using helminths to treat some of those diseases. This review focuses on the use of human hookworm Necator americanus as a treatment for some of the autoimmune and inflammatory diseases.

Keywords: Necator americanus, hookworm, autoimmune disease, inflammatory disease, treatment

\section{Introduction}

Human hookworm diseases are the important neglected tropical diseases caused by two soil-transmitted helminth species: Necator americanus and Ancylostoma duodenale. It is estimated that 1.5 billion people are exposed to soil-transmitted helminth infection incurring $24 \%$ of the world's population. ${ }^{1}$ Hookworm alone infects $~ 576-740$ million people worldwide. Hookworm infection occurs by penetration of stage 3 infective larvae (iL3) through skin. Following the larvae entering the bloodstream via capillaries, they travel to the lungs, migrating via the respiratory tract to the throat where they are swallowed prior to passing down the esophagus into the small intestine. It is here where the larvae feed, mature to adulthood, mate, and produce eggs. The eggs are released in the feces into the environment and, under favorable environmental conditions, they hatch and develop into infective larvae, completing the life cycle. According to Centers for Diseases Control and Prevention, most of the people infected with hookworms are asymptomatic, especially during the primary infection. High hookworm burden usually induces anemia and protein loss, with such infections easily treatable. ${ }^{2}$ There are, however, some notable differences between natural and experimental infections. In experimental hookworm infections, some patients report very mild diarrhea and abdominal discomfort 4 weeks postinfection, coinciding with the arrival of the immature stage of larvae in the small intestine. In natural infection, the number of infective larvae penetrating the skin or the length of infection is usually unknown. ${ }^{3,4}$

\section{Immunology of experimental hookworm infection}

Helminths are a very broad group of parasites, generating many similarities and differences in the host immune response. However, there are numerous studies 
involving experimental human hookworm infection, which helped to clarify some points relating to this infection. With respect to $N$. americanus, the current methodology for the determination of a hookworm infection is via the presence of eggs released in the feces. The majority of the studies report findings during or after the establishment of the patency.

During the 1980s, experimental hookworm infection studies in healthy patients started to elucidate the early events of infection. One of the pioneer studies evaluated the events following exposure of volunteers to 50 infective stage 3 larvae (iL3). This study proved that infection with up to 50 iL3 of $N$. americanus was safe and tolerable, with no anemia, associated with low infection rates. At week 5 postinfection, patients reported flatulence and painful events, with symptoms linked to the establishment of worms in the small intestine. Mucosal erythema in the bronchoalveolar lavage was also shown. Eosinophilia in the blood peaked between 38 days and 64 days postinfection (dpi). However, only a few patients had levels of antibodies (IgE and IgG) augmented, with levels of both immunoglobulins decreased after the infection was cleared. ${ }^{5}$

The parameters evaluated during infection with 50 iL3 varied among the studies. In 2005, a study demonstrated that the peak of egg production present in the stool occurred 145 days postinfection; however, its presence in the stool started as early as 7.5 weeks postinfection. Additionally, this study demonstrated that IgG and IgE sera levels increased during the patent phase. Parasite load was also evaluated and showed that volunteers with 50 iL3 infection had between 600 and 900 eggs of hookworm released per gram (epg) of stool. Analysis of the blood parameters, at the same evaluation day (42 dpi), showed an increase in the number of white blood cells and eosinophils by 2.5 and 5 times, respectively, when compared to prior infection. One of the major advantages of experimental infection is the possibility to follow those parameters normally difficult to evaluate, for example, the level of cytokines. This study demonstrated that infection with $N$. americanus induced T-helper cell type 2 (Th2)-type cytokines. Moreover, interleukin (IL)-5 was produced very early postinfection and peaked at $20 \mathrm{dpi}$, while IL-13 peaked at 84 dpi. Other cytokines, for example, IL-10 and interferon $\gamma($ IFN- $\gamma)$, were detected demonstrating that hookworm infection generates a complex and mixed immune response. Interestingly, there was a positive correlation between the levels of IL-5 detected in the blood and the presence of eggs in the feces. No correlation, either with the egg counts in the feces or with the eosinophils in the blood, was seen with IL-13. ${ }^{6}$

In vitro studies on peripheral blood mononuclear cells (PBMCs) cultured with hookworm antigens are used to amplify the response to those antigens. The following three major N. americanus groups of antigens are studied: iL3 extract, adult worm extract (AdEx or AE), and excreted/ secreted proteins from adult worms. It was seen that the antigens iL3 or adult worm extract increased the proliferation and the production of tumor necrosis factor (TNF) in PBMCs from experimentally infected people evaluated during patency and early infection time points. A decrease in the same group of cells' proliferation was seen during prepatency period, although TNF production remained increased. Other responses were also demonstrated: stimulation with iL3 antigen induced an increase in CCL17 chemokine production, while excreted/secreted proteins induced IL-10 production in PBMCs. ${ }^{7}$

\section{Hookworm infection for treating inflammatory and autoimmune disease}

According to the American Autoimmune Diseases Association (AAADA), there are $~ 80-100$ autoimmune diseases identified and another 40 diseases that have an autoimmune component. Many chronic diseases with inflammatory and destructive components were found to be autoimmune, including rheumatoid arthritis, Grave's disease, Hashimoto's thyroiditis, type 1 diabetes, and celiac disease. ${ }^{8}$ One hundred thirty-four autoimmune diseases were listed in the AAADA web site, with the National Institute of Health estimating that 24.5 million people suffer with these diseases in the US and $3 \%-5 \%$ of the population estimated as being affected. Since the number of diseases and the etiology are varied, one should additionally consider that autoimmune diseases can affect several parts of the body either isolated or simultaneously. Furthermore, the symptoms and the disease time course can vary among the disease states. ${ }^{9,10}$

From an immunological point of view, some diseases such as type 1 diabetes, rheumatoid arthritis, lupus, and multiple sclerosis have an important Th1 component. Thus, a predisposition to produce type 1 cytokines, or even virus or bacterial infection, could lead to an induction of those inflammatory and autoimmune diseases. On the contrary, helminth diseases have a strong Th2 or modulatory type of response and could contribute to balance the immune system. ${ }^{11}$

Autoimmune diseases are very complex, and a modest variation in the phenotype can have an impact on the disease and influence subsequent therapy. Due to the effect of the disease state on treatment, it is necessary to understand the genetic and immunological variances among the individual patients and the effect of early intervention and avoidance of complications. The disease percentage rates among identical 
twins can be very low in some diseases, for example, $15 \%$ in rheumatoid arthritis and $24 \%$ in systemic lupus erythematosus but slightly higher in type 1 diabetes with $30 \%$ coincidence in this population. ${ }^{8,12,13}$

Hookworm is emerging as a possible therapy for some autoimmune diseases. However, there are difficulties in using an animal experimental model for human hookworm since $N$. americanus is highly adapted to the human body, with mice not easily susceptible to infection. Hamsters can be infected; however, they are not the most suitable model due to the limited availability of species-specific reagents. Moreover, in the hamster model, there is a need for corticosteroid injections in conjunction with $N$. americanus to maintain the ongoing infection. Also, hamster hookworm infections are costly and time consuming with the recovered worms much smaller in size when compared with those in human infections. Worms in the human gut can survive several years, and if people maintain suitable hygiene standards, random infection is kept very low or nonexistent. ${ }^{14-16}$ Hence, for clinical trials of some autoimmune diseases, it is essential to use human hookworm infection as opposed to model systems.

In 2006, it was described that infection with 10, 25, 50, and 100 iL3 larvae was well tolerated, although itching on the skin entry site and gastrointestinal discomfort were more common in higher doses. The number of eggs recovered was at least $50 \mathrm{epg}$. Levels of eosinophilia, blood IgE (from week 6 postinfection), and $\operatorname{IgG}$ (weeks 8,10 , and 12 postinfection) were detected even in low iL3 infection rates. Additionally, there was no significant change in anemia, measured by the hemoglobin levels and hematocrit, demonstrating that ten $N$. americanus iL3 would be suitable for the therapy of inflammatory diseases. ${ }^{17,18}$ Moreover, basophilic activation was noted in very early infection (4 weeks) and was kept higher than placebo control at weeks 6 and 12 postinfection. However, this activation was not followed by an increase in hookworm IgE, confirming that an experimental hookworm infection using ten iL3 was still within safe monitoring parameters. $^{19}$

The way in which hookworm or other helminths could control an inflammatory or autoimmune disease is not yet clear. It is expected that different diseases elicit different immune responses in the presence of helminths. Even with similarities in the activated immune compartments associated with these diseases, there are differences in the helminths individual genome/proteome. Mechanisms of suppression of the inflammatory diseases rely on both adaptive and innate immune responses, including mechanisms of the worm evading the host response and the immunomodulatory effects associated with the infection as indicated by the presence of Th2, Treg, modulatory cytokines, and B-cells. ${ }^{20}$

To date, there are 41 clinical trials involving hookworm registered at www.clinicaltrials.gov database. Among them, only six clinical trials are evaluating hookworm as therapy for inflammatory diseases. Diseases that are currently being investigated or already completed and have examined the influence of hookworm in their progression are asthma (one clinical trial), allergic rhinoconjunctivitis (one clinical trial), multiple sclerosis (two clinical trials), and celiac disease (two clinical trials). ${ }^{21}$

This review is based on the information found in the published studies and the two available platforms PubMed and Google Scholar. Therefore, it is important to note that of the two multiple sclerosis clinical trials in www.clinicaltrials.gov database, one (NCT00630383) was withdrawn prior to enrolment in June 2012 due to it being superseded by another similar study (NCT01470521). This later study (NCT01470521) consisted of a double-blind Phase II trial with 72 patient enrollments, with the study set for completion in January 2016; the results of this trial are not yet available.

\section{Allergies and hookworm}

In 2001, Scrivener et al studied the effect of human hookworm ( $N$. americanus) infection in patients with wheeze, associated with a domestic allergen. There was a strong association between allergen wheeze and the house localization (urban area) in this study. In comparison, infection with hookworm was negatively associated with wheeze. ${ }^{22}$

In Vietnam, a study with 1,601 children aged between 6 years and 18 years evaluated the influence of helminth infection on skin sensitization. Infection with natural hookworm burden (>350 epg) was associated with reduced dust mites sensitization. ${ }^{23}$ In the same country, a study with 1,587 children from a rural area demonstrated that the decrease in the skin allergen sensitization was related to the presence of IL-10 produced against hookworm at baseline. Interestingly, there was no difference between cytokine production in the placebo group and hookworm drug-treated group. Hence, the results clearly demonstrate that an infection with hookworm reduces the risk of allergen skin sensitization. ${ }^{24,25}$

Of interest is a placebo-controlled trial in Uganda involving 2,507 pregnant women. Following the birth of the 2,345 children, 1,635 children were followed up for 5 years. It was observed that hookworm infection alone, not the presence of other helminths, was negatively associated with reduced eczema during mother's pregnancy and in the children. Hence, 
there was a strong association between eczema in mother and child when mothers were not infected with hookworm. Additionally, the presence of a cockroach-specific IgE in children was negatively associated with a pregnancy-associated hookworm infection. ${ }^{26}$ Hence, sensitization during pregnancy improved the response against eczema in children born from hookworm-infected mothers, demonstrating that hookworm has the possibility to control or improve skin allergy; however, the mechanisms involved require further elucidation.

Based on the studies working on natural hookworm infection, a clinical trial was conducted for 12 weeks evaluating patients with allergic rhinoconjunctivitis and patients concurrently infected with hookworm $N$. americanus. This trial shows that infection with ten iL3 was not capable of changing the levels of allergen-related $\operatorname{IgE}$ and the cytokine production of TNF, IL-4, and IL-10, when compared with placebo control. Although not significant, it should be noted that IFN- $\gamma$ levels decreased with the patency establishment as measured by the presence of eggs released in the feces at week 6 postinfection. ${ }^{18}$ These combined results indicate that the dose of hookworm infection needs to be adjusted, in a safe trial-and-error manner, to help to deliver an effective treatment for allergic rhinoconjunctivitis. Interestingly, patients with allergic rhinoconjunctivitis showed increased skin itchiness and redness compared with control group, particularly during 1 week postinfection. Additionally, some patients reported abdominal pain; however, patients infected with hookworm reported an improvement in hay fever symptoms. As a result, eleven of 13 participants decided not to terminate the infection following the conclusion of the clinical trial, with a further eleven people from placebo control group electing to be infected with hookworm. ${ }^{27}$

\section{Asthma and hookworm}

A randomized placebo-controlled trial in asthma disease was performed in 32 patients diagnosed and treated with corticosteroids (up to $1,000 \mathrm{mg}$ beclometasone). The trial was followed up for 16 weeks. Both primary and secondary outcomes showed no significant differences between the groups tested. Peripheral eosinophils rose $\sim$ day 21 post-hookworm infection, with the peak between 42 days and 84 days postinfection. At the end of the study, 13 in 16 patients $(>80 \%)$ with hookworm infection decided not to take antihelminth treatment, although no significant improvement in asthma measurements was seen. There are a few theories about the reasons why this trial did not succeed. Although there was a possibility that the association between hookworm infection and improvement of asthma was false, there was a hypothesis that the presence of corticosteroids treatment impairs or does not allow the immune system to respond accordingly during hookworm infection. Furthermore, on this trial, several participants did not release eggs in the feces; the only measurement able to prove current infection. ${ }^{28}$ This study also kept a very low dose of ten iL3 as infection rate; thus, the epg counting, when present, was up to 50, proving a very low infection burden. In wheeze measurements, clinical trials had indicated that patients with higher epg counts (48-1,458 epg) showed better protection. ${ }^{22}$ Indeed, more studies with higher hookworm infection safe doses should be performed to clarify the role of hookworm in asthma.

Therefore, the current infection with hookworm was associated with lower risk of asthma and a decreased effect on wheeze. Other helminths also showed similar response. Nonetheless, $N$. americanus was the only helminth to show dose-related infection intensity in the reduction of both asthma and wheeze. ${ }^{29}$

\section{Crohn's disease and hookworm}

Crohn's disease is one type of inflammatory bowel disease. One of the first studies involving hookworm infection and Crohn's disease was performed in Australia with a very small number of patients. In this study, Crohn's disease patients and researchers received inocula, where three received $25 \mathrm{iL3}$, five received $50 \mathrm{iL3}$, and one received 100 iL3. The iL3 strain was donated from UK and originally came from Madang, Papua, New Guinea. Penetration of iL3 through skin was reported to cause itchiness that disappeared after a few days in all patients. The patients reported no symptoms related to pulmonary or respiratory tract. Blood eosinophilia was prominent from 5 weeks postinfection and stayed high until week 20 postinfection when compared with preinfection rates. All the patients developed patent infection by week 20 after iL3 administration, and at this time point, Crohn's disease activity index was measured and showed an improvement. There was no statistical difference in hemoglobin during the whole infection period. Reinoculation was offered and performed in five of eight subjects. At week 27 from first infection, four patients previously infected with 25 iL3 received another 25 iL3, one previously infected with 50 iL3 had received another $50 \mathrm{iL3}$, and at week 30 , another patient previously infected with 50 iL 3 dose received 25 iL3. In two of the five patients, there was a disease reactivation by week 45 (counting from first infection), and three improved the Crohn's disease scores and reduced immunosuppression drugs. All 
five reinoculated patients were in remission by week 45 , indicating that hookworm had a summit effect on the immunosuppressive therapy, improving their health. ${ }^{30}$ Following this study, the same group of researchers also evaluated the arrival of the worms in the gut after reinoculation. The results demonstrated that either in Crohn's disease patients or in healthy infected people, the distribution of the worms in the gut followed the presence of current infection; that is, mature worms populated preferentially the distal duodenum and proximal jejunum. Moreover, immature worms were expelled distally. Together, these findings suggested that hookworm infection could benefit better, diseases that occur in the same location where mature worms reside. ${ }^{30}$

In 2011, a study from India was published with results from 78 Crohn's disease patients and 75 healthy control individuals. The majority of the Crohn's disease patients were from urban area (57 out of 78) but control individuals were evenly distributed among the areas. In regard to T-cell activation on hookworm crude extract, patients with Crohn's disease showed decreased percentage in the number of T-cells expressing CD69 than that in the healthy individuals. In response to hookworm antigens in vitro, 36 healthy people $(48 \%)$ and 20 Crohn's disease patients $(25 \%)$ produced IFN- $\gamma$ $(P=0.005)$. Taken together, T-cell activation, production of IFN- $\gamma$ in response to hookworm antigens, and residence in rural areas are inversely related to this disease. ${ }^{31}$ People with disease diagnosed for over 2 years were excluded, as well as people using immunosuppressant steroids or infliximab (antibody anti-TNF). ${ }^{32}$ It is probable that the worst cases were also excluded. Hence, this study also indicates that hygiene hypothesis needs further investigation, since it lacked specific markers for previous hookworm infection as enzyme-linked immunosorbent assay (ELISA) against worm antigens demonstrates cross-reactivity with other helminths. ${ }^{33}$

\section{Celiac disease and hookworm}

Celiac disease is an autoimmune disease in genetically susceptible people developed by a diet with gluten ingestion. In the absence of gluten ingestion, most of the celiac disease patients can control the disease and enter remission. ${ }^{34}$

The first clinical trial with celiac disease patients was based on a safe dose of hookworm infection (10+5 iL3) and a well-known gluten challenge ( $16 \mathrm{~g}$ of gluten a day for 5 days). On this trial, 20 patients were selected and divided into double-blind placebo (chili) control and infected groups. At week 0 , patients from the infected group received ten iL3, and at week 12, another five larvae. Blood was withdrawn at week 0 (baseline), 4, 12, 20 (pregluten challenge), and 21 (post-gluten challenge) postinfection. At weeks 20 and 21, duodenal biopsies were also collected to evaluate the damage in the gut. All ten patients from the infected group had infection confirmed either by worm visualization during endoscopy or by the presence of eggs in the feces. Although not statistically significant, it was notable that the patients in the infected group answered a better score for lethargy than control group. Blood eosinophilia was observed from week 4 in the infected group and stayed higher than control until week 21. After the gluten challenge intake, at week 21 , the percentage of T-cells producing IFN- $\gamma$ in response to QE65 gluten peptide in vitro was increased only for the placebo group, although the differences between the study groups were not significant. ${ }^{3}$

Additionally, after the gluten challenge, patients with hookworm infection demonstrated decreased levels of inflammatory cytokines IFN- $\gamma$ and IL-17A spontaneously produced by duodenal biopsies, indicating that hookworm infection could induce modulation in the inflammatory site. In response to QE65 gluten peptide, biopsies from infected individuals showed a strong IL-2, IL-17A, and IFN- $\gamma$ production. Interestingly, IL-5 production in response to QE65 stimulation was only seen in biopsies from the infected group. Together, the results demonstrate that the capacity of hookworm infection modulation would be via Th2 and IL-10, regulating Th1/Th17 response. Remarkably, from 20 initial patients, 17 decided to keep the infection believing that they could benefit in the long-term from it. ${ }^{35}$ This clinical trial also contributed to the understanding of hookworm infection since in the endemic areas we have no knowledge of parasite burden in the intestine, neither the time nor length of the infection. A systemic Th 2 response was observed, with an increase in the levels of IL-5 and IL-13 starting from week 4 and peaking at week 12 postinfection. At the parasite site, the intestinal biopsies showed increased levels of IFN- $\gamma$ mRNA after the infection compared with the levels of IFN- $\gamma$ mRNA before the infection. Since a Th1 profile was not observed, the presence of IFN- $\gamma$ was considered to most likely be from an innate source. Furthermore, in this study, healthy celiac patients (before gluten challenge) also showed that mRNA levels of ALDH1A2 and IL-15 were increased after infection, although there was a decrease in the mRNA levels of IL-23 when comparing postinfection versus pre-hookworm infection. The biopsies of the celiac patients were then restimulated with excretory/secretory proteins from adult worms. The result was a strong Th2 response with increased mRNA levels of IL-4, IL-5, IL-13, IL-9, and IL-10. This effect was also shown in PBMCs under the same restimulation conditions. Levels of 
IL-15, transforming growth factor $\beta$, and IL-22 mRNA were also increased in biopsies cultured with excretory/secretory proteins. Thus, it was hypothesized that current hookworm infection can decrease the levels of proinflammatory cytokines and immune cell activation, inducing a strong Th2 and regulatory response environment that will contribute to the worm survival for many years in the host. ${ }^{36}$

Nevertheless, it should be noted that the number of infective larvae used, although very safe, was also very low, considering that $16 \mathrm{~g}$ of gluten a day for 5 days is a large amount of gluten with which to challenge celiac disease patients. Thus, another question rose from this study: would it be possible to induce a gluten tolerance in the patients infected with hookworm?

Another study was recently conducted involving 12 celiac disease patients, evaluating the clinical trial for 52 weeks. At this time point of assessment, the subjects received $20 \mathrm{iL3}$, ten at week 0 and ten at weeks $4-8$. Gluten challenge was performed for all of the patients as follows: microchallenge $(10-50 \mathrm{mg}$ of gluten for 12 weeks), GC-1g group ( $25 \mathrm{mg}$ daily plus $1 \mathrm{~g}$ for 12 weeks), and GC-3g (3 g of gluten for 2 weeks). Unfortunately, not all subjects completed the trial; two subjects needed to withdraw after microchallenge, ten completed the $1 \mathrm{~g}$ challenge and eight completed the $3 \mathrm{~g}$ challenge, showing that there are still some unpredictable effects that need to be clarified. No signs of anemia and classical eosinophilia were observed. The results of this trial showed that there were no differences in the gut villi crypt depth measurements after $1 \mathrm{~g}$ challenge. Antiglutaminase IgA titers declined after $1 \mathrm{~g}$ challenge but increased after $3 \mathrm{~g}$ challenge. The presence of IFN- $\gamma$ producing intestinal T-cells decreased and regulatory T-cell (CD4+FoxP3+) increased after hookworm infection. Thus, hookworm infection together with microchallenges showed promotion of tolerance and improvement of the participants' quality of life. ${ }^{37}$ The patients also had their gut microbiota evaluated. In patients infected with hookworm, the gut flora composition did not change after the gluten challenge. Additionally, there was an increase in the number of species present in the gut of hookworm-infected people through the clinical trial indicating that the maintenance of gut microflora richness could be a way of regulating the celiac disease via hookworm infection. How this relationship works and flows still needs more elucidations. ${ }^{3840}$

\section{Final remarks}

Very light hookworm infection was proven to be safe, especially in the developed countries where good sanitation and hygiene habits are not a problem. Considering the power of hookworm infection in the induction of Th2 and/or modulatory response, very little has been studied with regard to using infection to control autoimmune and inflammatory diseases. It is also necessary to keep in mind that autoimmune and inflammatory diseases have a broad spectrum of symptoms, treatment, and varying sites of injury. However, with the increasing number of these diseases, there is a need for new and intelligent interventions for the early prevention and control of them. It is also necessary to point out that not all patients would feel comfortable receiving a worm infection, especially because of the lack of knowledge about the parasite they have. With the advantage of bioinformatics and published parasites' genomes, the science community should benefit and start looking for possible modulatory molecules to treat inflammatory and autoimmune diseases, considering the particularities of each parasite and disease of interest.

\section{Acknowledgments}

Dr Gaze is supported by CNPq Universal Grant (444060/201417) and Oswaldo Cruz Foundation. Dr Gaze thanks Leanne Cooper and Rodrigo Jangola for English language review of this manuscript.

\section{Disclosure}

The author reports no conflicts of interest in this work.

\section{References}

1. World Health Organization [webpage on the Internet]. Hookworm Disease; 2012. Available from: http://www.who.int/vaccine_research/ diseases/soa_parasitic/en/index2.html. Accessed May 11, 2016.

2. CDC [webpage on the Internet]. Hookworm; 2013. Available from: http://www.cdc.gov/parasites/hookworm/. Accessed November 12, 2015 .

3. Daveson AJ, Jones DM, Gaze S, et al. Effect of hookworm infection on wheat challenge in celiac disease - a randomised double-blinded placebo controlled trial. PLoS One. 2011;6(3):e17366.

4. Gaze S, Bethony JM, Periago MV. Immunology of experimental and natural human hookworm infection. Parasite Immunol. 2014;36(8): 358-366.

5. Maxwell C, Hussain R, Nutman TB, et al. The clinical and immunologic responses of normal human volunteers to low dose hookworm (Necator americanus) infection. Am J Trop Med Hyg. 1987;37(1):126-134.

6. Wright V, Bickle Q. Immune responses following experimental human hookworm infection. Clin Exp Immunol. 2005;142(2):398-403.

7. Geiger SM, Fujiwara RT, Santiago H, Corrêa-Oliveira R, Bethony JM. Early stage-specific immune responses in primary experimental human hookworm infection. Microbes Infect. 2008;10(14-15):1524-1535.

8. Cho JH, Feldman M. Heterogeneity of autoimmune diseases: pathophysiologic insights from genetics and implications for new therapies. Nat Med. 2015;21(7):730-738.

9. Jacobson DL, Gange SJ, Rose NR, Graham NM. Epidemiology and estimated population burden of selected autoimmune diseases in the United States. Clin Immunol Immunopathol. 1997;84(3):223-243.

10. NIH [webpage on the Internet]. Understanding Autoimmune Diseases; 2015. Available from: http://www.niams.nih.gov/Health_Info/Autoimmune/. Accessed November 12, 2015.

11. Marrack P, Kappler J, Kotzin BL. Autoimmune disease: why and where it occurs. Nat Med. 2001;7(8):899-905. 
12. Roep BO, Buckner J, Sawcer S, Toes R, Zipp F. The problems and promises of research into human immunology and autoimmune disease. Nat Med. 2012;18(1):48-53.

13. Bogdanos DP, Smyk DS, Rigopoulou EI, et al. Twin studies in autoimmune disease: genetics, gender and environment. J Autoimmun. 2012; 38(2-3):J156-J169.

14. Rajasekariah GR, Dhage KR, Deb BN, Bose S. Necator americanus and Ancylostoma ceylanicum: development of protocols for dual infection in hamsters. Acta Trop. 1985;42(1):45-54.

15. Behnke JM, Pritchard DI. Necator americanus in neonatally infected hamsters. The time-course of infection and antibody response to the surface antigens of L4 and adult worms. Trans R Soc Trop Med Hyg. 1987; 81(6):967-972.

16. Fujiwara RT, Geiger SM, Bethony J, Mendez S. Comparative immunology of human and animal models of hookworm infection. Parasite Immunol. 2006;28(7):285-293.

17. Mortimer K, Brown A, Feary J, et al. Dose-ranging study for trials of therapeutic infection with Necator americanus in humans. Am J Trop Med Hyg. 2006;75(5):914-920.

18. Blount D, Hooi D, Feary J, et al. Immunologic profiles of persons recruited for a randomized, placebo-controlled clinical trial of hookworm infection. Am J Trop Med Hyg. 2009;81(5):911-916.

19. Falcone FH, Telford G, Hooi D, et al. Antigen-driven basophil activation is indicative of early Necator americanus infection in IgE-seronegative patients. J Allergy Clin Immunol. 2009;124(6):1343.e7-1350.e7.

20. McSorley HJ, Hewitson JP, Maizels RM. Immunomodulation by helminth parasites: defining mechanisms and mediators. Int J Parasitol. 2013;43(3-4):301-310.

21. NIH. Clinical trials. gov [homepage on the Internet]. Available from: https://clinicaltrials.gov/. Accessed November 4, 2015.

22. Scrivener S, Yemaneberhan H, Zebenigus $M$, et al. Independent effects of intestinal parasite infection and domestic allergen exposure on risk of wheeze in Ethiopia: a nested case-control study. Lancet. 2001;358(9292): 1493-1499.

23. Flohr C, Tuyen LN, Lewis S, et al. Poor sanitation and helminth infection protect against skin sensitization in Vietnamese children: a crosssectional study. J Allergy Clin Immunol. 2006;118(6):1305-1311.

24. Flohr C, Tuyen LN, Quinnell RJ, et al. Reduced helminth burden increases allergen skin sensitization but not clinical allergy: a randomized, doubleblind, placebo-controlled trial in Vietnam. Clin Exp Allergy. 2010;40(1): 131-142.

25. Feary J, Britton J, Leonardi-Bee J. Atopy and current intestinal parasite infection: a systematic review and meta-analysis. Allergy. 2011;66(4): $569-578$.
26. Mpairwe H, Ndibazza J, Webb EL, et al. Maternal hookworm modifies risk factors for childhood eczema: results from a birth cohort in Uganda. Pediatr Allergy Immunol. 2014;25(5):481-488.

27. Feary J, Venn A, Brown A, et al. Safety of hookworm infection in individuals with measurable airway responsiveness: a randomized placebo-controlled feasibility study. Clin Exp Allergy. 2009;39(7): 1060-1068.

28. Feary JR, Venn AJ, Mortimer K, et al. Experimental hookworm infection: a randomized placebo-controlled trial in asthma. Clin Exp Allergy. 2010;40(2):299-306.

29. Leonardi-Bee J, Pritchard D, Britton J. Asthma and current intestinal parasite infection: systematic review and meta-analysis. Am J Respir Crit Care Med. 2006;174(5):514-523.

30. Croese J, O'neil J, Masson J, et al. A proof of concept study establishing Necator americanus in Crohn's patients and reservoir donors. Gut 2006;55(1):136-137.

31. Kabeerdoss J, Pugazhendhi S, Subramanian V, Binder HJ, Ramakrishna BS. Exposure to hookworms in patients with Crohn's disease: a case-control study. Aliment Pharmacol Ther. 2011;34(8):923-930.

32. Ramakrishna BS, Binder HJ, Subramanian V, Pugazhendhi S, Kabeerdoss J. Exposure to hookworms in patients with Crohn's disease: authors' reply. Aliment Pharmacol Ther. 2011;34(10):1250.

33. Shale M, Travis SP. Exposure to hookworms in patients with Crohn's disease. Aliment Pharmacol Ther. 2011;34(10):1248-1249.

34. Moore JK, West SR, Robins G. Advances in celiac disease. Curr Opin Gastroenterol. 2011;27(2):112-118.

35. McSorley HJ, Gaze S, Daveson J, et al. Suppression of inflammatory immune responses in celiac disease by experimental hookworm infection. PLoS One. 2011;6(9):e24092.

36. Gaze S, McSorley HJ, Daveson J, et al. Characterising the mucosal and systemic immune responses to experimental human hookworm infection. PLoS Pathog. 2012;8(2):e1002520.

37. Croese J, Giacomin P, Navarro S, et al. Experimental hookworm infection and gluten microchallenge promote tolerance in celiac disease. J Allergy Clin Immunol. 2015;135(2):508-516.

38. Cantacessi C, Giacomin P, Croese J, et al. Impact of experimental hookworm infection on the human gut microbiota. J Infect Dis. 2014 210(9):1431-1434.

39. Giacomin P, Zakrzewski M, Croese J, et al. Experimental hookworm infection and escalating gluten challenges are associated with increased microbial richness in celiac subjects. Sci Rep. 2015;5:13797.

40. Giacomin P, Croese J, Krause L, Loukas A, Cantacessi C. Suppression of inflammation by helminths: a role for the gut microbiota? Philos Trans $R$ Soc Lond B Biol Sci. 2015; 370(1675):20140296.
Reports in Parasitology

\section{Publish your work in this journal}

Reports in Parasitology is international, peer-reviewed, open access journal publishing original research, reports, reviews and commentaries on all areas of parasitology. The manuscript management system is completely online and includes a very quick and fair peer-review system. Visit http://www.dovepress.com/testimonials.php to read real quotes from published authors.

\section{Dovepress}

